BULL. AUSTRAL. MATH. SOC.

60005,53065

VOL. 19 (1978), 145-146.

\title{
Stereology - a statistical viewpoint
}

\section{Pamela Joy Davy}

Various geometrical properties of a feature set contained within a compact $n$-dimensional specimen $X$ may be inferred by generating a random position of a probe intersecting $X$ or by projecting $X$ in an isotropic random direction. The variance associated with such sampling is highly structure dependent and may be expressed in terms of a double integral over $X$. Ratio estimation, in the form of a set of fundamental formulae, is widely used in stereology, and is shown to be unbiased with the use of appropriately weighted probes, which are relatively easy to generate in a geometrical setting. Certain advantages other than elimination of bias are inherent in weighted sampling - in particular the mean square error is, in certain circumstances, reduced. Besides providing a rigorous derivation (which, except under very restricted conditions, has been lacking even for 2 or 3 dimensions), this thesis provides new stereological formulae of potential application. Variants of standard sectioning, namely wedge sections and curved sections, are also considered, with the interesting outcome that some properties not accessible by flat sectioning are thereby estimable.

Another interpretation of stereology is given in terms of random sets of infinite extent. The fundamental formulae in this case become identities between certain densities, which can be defined both as expected values of geometrical quantities per unit volume and as coefficients associated with the intersection of a fixed compact convex set with the random set.

One practical problem in transmission microscopy is that of correcting the fundamental formulae when a slice of non-zero thickness is observed

Received 17 March 1978. Thesis submitted to the Australian National University, March 1978. Degree approved, September 1978. Supervisor: Dr Roger E. líles. 
instead of a true section. A multi-dimensional treatment of this problem is given. Multiple sampling, either systematic or independent, is important to increase the precision of stereological estimates. Multistage procedures, which can be formulated in terms of martingales, are also investigated. Certain techniques of Monte Carlo estimation have a special interpretation in the context of stereology. 